\title{
A TIGHTNESS PROPERTY OF A SYMMETRIC MARKOV PROCESS AND THE UNIFORM LARGE DEVIATION PRINCIPLE
}

\author{
MASAYOSHI TAKEDA
}

\author{
(Communicated by Edward C. Waymire)
}

\begin{abstract}
Previously, we considered a large deviation for occupation measures of a symmetric Markov processes under the condition that its resolvent possesses a kind of tightness property. In this paper, we prove that if the Markov process is conservative, then the tightness property implies the uniform hyper-exponential recurrence, which leads us to the uniform large deviation principle.
\end{abstract}

\section{INTRODUCTION}

Let $E$ be a locally compact separable metric space and $m$ a positive Radon measure on $E$ with full support. Let $X=\left(\Omega, X_{t}, \mathbb{P}_{x}, \zeta\right)$ be an $m$-symmetric Borel right process on $E$. Here $\zeta$ is the lifetime of $X$. We assume that the process $X$ is irreducible and strong Feller. Moreover, we assume that $X$ possesses a tightness property; i.e., for any $\epsilon>0$, there exists a compact set $K$ such that $\sup _{x \in E} R_{1} 1_{K^{c}}(x) \leq \epsilon$. Here $1_{K^{c}}$ is the indicator function of the complement of $K$ and $R_{1}$ is the 1-resolvent of $X$. In [18, [19], we consider large deviations for empirical measures of symmetric Markov processes with the tightness property.

We prove in this note that if $X$ is conservative, $\mathbb{P}_{x}(\zeta=\infty)=1$, then the tightness property implies the positive recurrence of $X$; in particular, the measure $m$ turns out to be finite. Moreover, we prove that if, in addition, there exists an increasing sequence $\left\{K_{n}\right\}_{n=1}^{\infty}$ of compact sets such that the union of $\left\{K_{n}\right\}_{n=1}^{\infty}$ equals $E$ and each part (absorbing) process $X^{D_{n}}$ on $D_{n}\left(D_{n}:=K_{n}^{c}\right)$ is irreducible, then $X$ possesses the following strong recurrence property: for any positive constant $\gamma$, there exists a compact set $K \subset E$ such that

$$
\sup _{x \in E} \mathbb{E}_{x}\left(\exp \left(\gamma \sigma_{K}\right)\right)<\infty,
$$

where $\sigma_{K}$ is the first hitting time of $K, \sigma_{K}=\inf \left\{t>0: X_{t} \in K\right\}$. Wu [21] calls this property a uniform hyper-exponential recurrence, and we prove that the property implies the uniform large deviation principle (Theorem 2.3 and Theorem 3.12 below). As an example, a one-dimensional diffusion process satisfies the uniform hyper-exponential recurrence, and thus the uniform large deviation principle, if

Received by the editors November 1, 2011 and, in revised form, February 13, 2012.

2010 Mathematics Subject Classification. Primary 60F10; Secondary 60J45, 31C25.

Key words and phrases. Large deviation, symmetric Markov process, Dirichlet form.

The author was supported in part by Grant-in-Aid for Scientific Research No. 22340024 (B), Japan Society for the Promotion of Science. 
both boundaries are an entrance in Feller's classification of the boundaries (Example 3.1). On the other hand, we see that if $X$ is not conservative, the tightness property implies a fast explosion in the sense that the lifetime $\zeta$ is exponentially integrable: for some $\gamma>0$,

$$
\sup _{x \in E} \mathbb{E}_{x}(\exp (\gamma \zeta))<\infty .
$$

There exist two key items in the proof of these facts: one is an inequality due to Stollman and Voigt (see (2.6)), and the other is the identification of DonskerVaradhan's I-function (see (2.5)) with the Dirichlet form (Proposition 2.4). Combining these facts with the tightness property, we can show that the subset of probability measures on $E$ defined by $\left\{u^{2} \cdot m: \int_{E} u^{2} d m=1, \mathcal{E}(u, u) \leq l\right\}, l>0$, is compact with respect the weak topology, which leads us to the existence of the ground state (Lemma 2.6). Here $\mathcal{E}$ is the Dirichlet form generated by $X$ (see (2.1)).

We finally discuss sufficient conditions for a part process on an open set to be irreducible, because this property is needed for the proof of the uniform hyperexponential recurrence (Remark 3.7, Lemma 3.9).

\section{Existence of the Ground state}

Let $E$ be a locally compact separable metric space, $E_{\Delta}=E \cup\{\Delta\}$ the one point compactification of $E$, and $m$ a positive Radon measure on $E$ with full support. Let $X=\left(\Omega, \mathcal{F},\left\{\mathcal{F}_{t}\right\}_{t \geq 0}, X_{t}, \mathbb{P}_{x}, \zeta\right)$ be an $m$-symmetric Borel right process having left limits on $(0, \zeta)$. Here $\zeta$ is the lifetime $\zeta(\omega)=\inf \left\{s \geq 0: X_{s}(w)=\Delta\right\}$ and $\left\{\mathcal{F}_{t}\right\}_{t \geq 0}$ is the minimal (augmented) admissible filtration.

Let $\left\{p_{t}\right\}_{t \geq 0}$ be the semigroup of $X, p_{t} f(x)=\mathbb{E}_{x}\left(f\left(X_{t}\right)\right)$. By Lemma 1.4.3 in [7], $\left\{p_{t}\right\}_{t \geq 0}$ uniquely determines a strongly continuous Markovian semigroup $\left\{T_{t}\right\}_{t \geq 0}$ on $L^{2}(E ; m)$. We define the Dirichlet form $(\mathcal{E}, \mathcal{D}(\mathcal{E}))$ on $L^{2}(E ; m)$ generated by $X$ :

$$
\left\{\begin{array}{l}
\mathcal{D}(\mathcal{E})=\left\{u \in L^{2}(E ; m): \lim _{t \rightarrow 0} \frac{1}{t}\left(u-T_{t} u, u\right)_{m}<\infty\right\}, \\
\mathcal{E}(u, v)=\lim _{t \rightarrow 0} \frac{1}{t}\left(u-T_{t} u, v\right)_{m} .
\end{array}\right.
$$

We know that the Dirichlet form $(\mathcal{E}, \mathcal{D}(\mathcal{E}))$ is quasi-regular $([12])$.

A set $B \subset E_{\Delta}$ is said to be nearly Borel if for any probability measure $\mu$ on $E_{\Delta}$ there exist Borel sets $B_{1}, B_{2}$ of $E_{\Delta}$ such that $B_{1} \subset B \subset B_{2}$ and

$$
\mathbb{P}_{\mu}\left(X_{t} \in B_{2} \backslash B_{1}, \exists t \geq 0\right)=0 .
$$

A set $N \subset E$ is said to be m-polar if there exists a nearly Borel set $\widetilde{N} \subset E$ such that $N \subset \widetilde{N}$ and $\mathbb{P}_{m}\left(\sigma_{\widetilde{N}}<\infty\right)=0$. A statement depending on $x \in A$ is said to hold q.e. on $A$ if there exists an $m$-polar set $N \subset A$ such that the statement is true for every $x \in A \backslash N$ ("q.e." is an abbreviation of "quasi-everywhere").

Let us denote by $\left\{R_{\alpha}\right\}_{\alpha>0}$ the resolvent of $X$,

$$
R_{\alpha} f(x)=\mathbb{E}_{x}\left(\int_{0}^{\infty} e^{-\alpha t} f\left(X_{t}\right) d t\right), \quad f \in \mathcal{B}_{b}(E)
$$

where $\mathcal{B}_{b}(E)$ is the space of bounded Borel functions on $E$. We now introduce three properties of Borel right processes:

I. (Irreducibility) If a Borel set $A$ is $p_{t}$-invariant, i.e., $\int_{A} p_{t} 1_{A^{c}} d m=0$ for any $t>0$, then $A$ satisfies either $m(A)=0$ or $m\left(A^{c}\right)=0$. Here $1_{A^{c}}$ is the indicator function of the complement of $A$. 
II. (Strong Feller Property) $p_{t}\left(\mathcal{B}_{b}(E)\right) \subset C_{b}(E), t>0$, where $C_{b}(E)$ is the space of bounded continuous functions.

III. (Tightness Property) For any $\epsilon>0$, there exists a compact set $K$ such that $\sup _{x \in E} R_{1} 1_{K^{c}}(x) \leq \epsilon$.

Here we make remarks on the tightness property.

Remark 2.1. (i) If the measure $m$ is finite, $m(E)<\infty$, and $\left\|R_{1}\right\|_{1, \infty}<\infty$, then $\left\|R_{1} 1_{K^{c}}\right\|_{\infty} \leq\left\|R_{1}\right\|_{1, \infty} m\left(K^{c}\right)$ and property III is fulfilled. Here $\left\|R_{1}\right\|_{1, \infty}$ is the operator norm from $L^{1}(E ; m)$ to $L^{\infty}(E ; m)$.

(ii) If $R_{1} 1 \in C_{\infty}(E)$, then $X$ is explosive and has property III. In fact, we have

$$
\sup _{x \in E} R_{1} 1_{K^{c}}(x)=\sup _{x \in K^{c}} R_{1} 1_{K^{c}}(x) \leq \sup _{x \in K^{c}} R_{1} 1(x) .
$$

Here $C_{\infty}(E)$ is the set of continuous functions vanishing at infinity. If $X$ is a diffusion process generated by a locally elliptic operator, the property that $R_{1} 1 \in$ $C_{\infty}(E)$ implies the compactness of $R_{1}$ as an operator on $L^{\infty}(E ; m)$, as a result, on $L^{2}(E ; m)($ [5, Theorem 6.1]).

(iii) If $C_{\infty}(E)$ is invariant under $R_{1}, R_{1}\left(C_{\infty}(E)\right) \subset C_{\infty}(E)$, then $R_{1} 1 \in C_{\infty}(E)$ is equivalent to property III. In fact, for a compact set $K$, take a positive function $g \in C_{\infty}(E)$ such that $1_{K} \leq g$. We then see from the invariance of $C_{\infty}(E)$ that $0 \leq \lim _{x \rightarrow \infty} R_{1} 1_{K}(x) \leq \lim _{x \rightarrow \infty} R_{1} g(x)=0$. Hence for any $\epsilon>0$ there exists a compact set $K$ such that

$$
\limsup _{x \rightarrow \infty} R_{1} 1(x) \leq \limsup _{x \rightarrow \infty} R_{1} 1_{K}(x)+\limsup _{x \rightarrow \infty} R_{1} 1_{K^{c}}(x) \leq \sup _{x \in E} R_{1} 1_{K^{c}}(x) \leq \epsilon,
$$

which implies $R_{1} 1 \in C_{\infty}(E)$. Hence, if $C_{\infty}(E)$ is invariant under $R_{1}$ and $X$ is conservative, $p_{t} 1=1$, then $X$ does not have the tightness property; in particular, the Ornstein-Uhlenbeck process does not.

(iv) If the Markov process $X$ is conservative, then property III implies that $X$ is positive recurrent (Lemma 3.2).

It follows from property II that the transitions probability $p_{t}(x, d y)$ is absolutely continuous with respect to $m$ :

$$
p_{t}(x, d y)=p_{t}(x, y) m(d y) \text { for each } t>0, x \in E .
$$

As a result, the resolvent kernel is also absolutely continuous with respect to $m$ : $R_{\beta}(x, d y)=R_{\beta}(x, y) m(d y)$. By [7, Lemma 4.2.4] the density $R_{\beta}(x, y)$ is assumed to be a non-negative Borel function such that $R_{\beta}(x, y)$ is symmetric and $\beta$-excessive in $x$ and in $y$. Under the absolute continuity condition, "quasi-everywhere" statements are strengthened to "everywhere" ones.

A positive measure $\mu$ is said to be smooth if there exists a positive continuous additive functional $A$ of $X$ such that for any positive Borel function $f$ and $\gamma$ excessive function $h(\gamma \geq 0)$, that is, $e^{-\gamma t} p_{t} h \leq h$,

$$
\lim _{t \rightarrow 0} \frac{1}{t} \mathbb{E}_{h \cdot m}\left[\int_{0}^{t} f\left(X_{s}\right) d A_{s}\right]=\int_{X} f(x) h(x) \mu(d x) .
$$

Here, $\mathbb{E}_{h \cdot m}[\cdot]=\int_{X} \mathbb{E}_{x}[\cdot] h(x) m(d x)$.

Following Z.-Q. Chen [1, we introduce classes of potentials. 
Definition 2.1. A positive smooth measure $\mu$ is said to be in the class $\mathcal{K}_{\infty}$ if for any $\epsilon>0$ there exist a compact subset $K$ and a positive constant $\delta>0$ such that for all measurable sets $B \subset K$ with $\mu(B)<\delta$,

$$
\sup _{x \in E} \int_{K^{c} \cup B} R_{1}(x, y) \mu(d y) \leq \epsilon .
$$

Under the condition for $X$ being transient, the class $\mathcal{K}_{\infty}$ is usually defined by using the Green kernel, i.e., the 0-resolvent density, and a measure $\mu$ in the class is said to be Green-tight. Here we use the 1-resolvent density to deal with recurrent processes. The next lemma is proven by Z.-Q. Chen ([1, Theorem 4.2]). We give a proof for completion.

Lemma 2.2. If $X$ satisfies II and III, then the measure $m$ belongs to $\mathcal{K}_{\infty}$.

Proof. By the definition of property III, there exists a compact set $K$ such that $\sup _{x \in E} \int_{K^{c}} R_{1}(x, y) \mu(d y) \leq \epsilon / 2$. Suppose that for any $\delta>0$ there exists a Borel set $B \subset K$ with $m(B) \leq \delta$ such that $\sup _{x \in E} R_{1} 1_{B}(x)>\epsilon / 2$. Then there exists a sequence $\left\{B_{n}\right\}_{n=1}^{\infty}$ of Borel subsets of $K$ such that $m\left(B_{n}\right) \leq 1 / 2^{n}$ and $\sup _{x \in K} R_{1} 1_{B_{n}}(x)>\epsilon / 2$. Define $A_{n}=\bigcup_{k=n}^{\infty} B_{k}$. Then $m\left(A_{n}\right)$ is less than $1 / 2^{n-1}$ and decreasingly converges to zero as $n \rightarrow \infty$. Hence $R_{1} 1_{A_{n}}$ decreasingly converges to zero point-wise. Since $R_{1} 1_{A_{n}}$ is continuous by the property II, $R_{1} 1_{A_{n}}$ uniformly converges to zero on $K$. This is contradictory to $\sup _{x \in K} R_{1} 1_{A_{n}}(x) \geq$ $\sup _{x \in K} R_{1} 1_{B_{n}}(x)>\epsilon / 2$.

We denote by $\mathcal{P}$ the set of probability measures on $E$. Define the function $I_{\mathcal{E}}$ on $\mathcal{P}$ of probability measures on $E$ by

$$
I_{\mathcal{E}}(\nu)= \begin{cases}\mathcal{E}(\sqrt{f}, \sqrt{f}) & \text { if } \nu=f \cdot m, \sqrt{f} \in \mathcal{D}(\mathcal{E}), \\ \infty & \text { otherwise. }\end{cases}
$$

The space $\mathcal{P}$ is supposed to be equipped with the weak topology. Given $\omega \in \Omega$ with $0<t<\zeta(\omega)$, let $L_{t}(\omega) \in \mathcal{P}$ be the normalized occupation distribution: for a Borel set $A$ of $E$,

$$
L_{t}(\omega)(A)=\frac{1}{t} \int_{0}^{t} 1_{A}\left(X_{s}(\omega)\right) d s .
$$

We proved the next theorem in $[18$.

Theorem 2.3. Assume that $X$ satisfies $I-I I I$.

(i) For each open set $G \subset \mathcal{P}$,

$$
\liminf _{t \rightarrow \infty} \frac{1}{t} \log \mathbb{P}_{x}\left(L_{t} \in G, t<\zeta\right) \geq-\inf _{\nu \in G} I_{\mathcal{E}}(\nu) .
$$

(ii) For each closed set $K \subset \mathcal{P}$,

$$
\limsup _{t \rightarrow \infty} \frac{1}{t} \log \sup _{x \in E} \mathbb{P}_{x}\left(L_{t} \in K, t<\zeta\right) \leq-\inf _{\nu \in K} I_{\mathcal{E}}(\nu) .
$$

We define the function space $\mathcal{D}^{+}$by

$$
\mathcal{D}^{+}=\left\{R_{\alpha} f: \alpha>0, f \in L^{2}(E ; m) \cap C_{b}^{+}(E) \text { and } f \not \equiv 0\right\},
$$

where $C_{b}^{+}(E)$ denotes the set of non-negative bounded continuous functions. We see that any function in $\mathcal{D}^{+}(A)$ is strictly positive by the irreducibility I. Define the 
operator $A$ on $\mathcal{D}^{+}$by $A R_{\alpha} f=\alpha R_{\alpha} f-f$ and the function $I$ on $\mathcal{P}$ by

$$
I(\nu)=-\inf _{\substack{u \in \mathcal{D}^{+} \\ \epsilon>0}} \int_{E} \frac{A u}{u+\epsilon} d \nu .
$$

The function $I$ is a version of the Donsker-Varadhan $I$-function introduced in [6]. Note that since the Markov process $X$ is allowed to have a finite lifetime, the function $u=R_{\alpha} f \in \mathcal{D}^{+}$is not alway uniformly lower-bounded by a positive constant even if $f$ is so, and consequently the function $A u / u$ is not always bounded. By adding a positive constant $\epsilon$, the function $A u /(u+\epsilon)$ is bounded continuous, and consequently the $I$-function defined by (2.5) is lower semicontinuous on $\mathcal{P}$ with respect to the weak topology. This is a reason why we modify the Donsker-Varadhan $I$-function. In spite of this modification, we can identify the $I$-function with the Dirichlet form ([7, Theorem 6.4.2]):

\section{Proposition 2.4.}

$$
I(\nu)=I_{\mathcal{E}}(\nu), \quad \nu \in \mathcal{P} .
$$

We define the subset $\mathcal{P}_{M}$ of $\mathcal{P}$ by

$$
\mathcal{P}_{M}=\left\{u^{2} \cdot m: u \in \mathcal{D}(\mathcal{E}), \int_{E} u^{2} d m=1, \mathcal{E}(u, u) \leq M\right\}, \quad M>0 .
$$

Lemma 2.5. The set $\mathcal{P}_{M}$ is compact in $\mathcal{P}$.

Proof. Recall the inequality in [14]: for any $\beta>0$ and any smooth measure $\mu$,

$$
\int_{E} u^{2}(x) \mu(d x) \leq\left\|R_{\beta} \mu\right\|_{\infty} \cdot\left(\mathcal{E}(u, u)+\beta \int_{E} u^{2} d m\right), \quad u \in \mathcal{D}(\mathcal{E}) .
$$

Combining property III with this inequality, we see that $\mathcal{P}_{M}$ is tight. Indeed, for any compact set $K \subset E$ and any $u^{2} \cdot m \in \mathcal{P}_{M}$,

$$
\int_{K^{c}} u^{2} d m \leq\left\|R_{1} 1_{K^{c}}\right\|_{\infty} \cdot\left(\mathcal{E}(u, u)+\int_{E} u^{2} d m\right) \leq(M+1)\left\|R_{1} 1_{K^{c}}\right\|_{\infty} .
$$

Since $\mathcal{P}_{M}=\{\nu \in \mathcal{P}: I(\nu) \leq M\}$ is closed by the lower semicontinuity of $I$, we have the lemma.

Let $\lambda_{2}$ be the bottom of the spectrum:

$$
\lambda_{2}=\inf \left\{\mathcal{E}(f, f): f \in \mathcal{D}(\mathcal{E}), \int_{E} f^{2} d m=1\right\} .
$$

A function $\phi_{0}$ on $E$ is called a ground state of the $L^{2}$-generator for $\mathcal{E}$ if $\phi_{0} \in$ $\mathcal{D}(\mathcal{E}),\left\|\phi_{0}\right\|_{2}=1$ and $\mathcal{E}\left(\phi_{0}, \phi_{0}\right)=\lambda_{2}$.

Lemma 2.6 ([19]). Assume that $X$ satisfies I-III. Then there exists a ground state $\phi_{0}$ uniquely up to a sign. $\phi_{0}$ can be taken to be strictly positive on $E$.

Proof. Let $\left\{u_{n}\right\}_{n=1}^{\infty} \subset \mathcal{D}(\mathcal{E})$ be a minimizing sequence, $\left\|u_{n}\right\|_{2}=1$, and $\lambda_{2}=$ $\lim _{n \rightarrow \infty} \mathcal{E}\left(u_{n}, u_{n}\right)$. We see from Lemma 2.5 that there exists a subsequence $\left\{u_{n_{k}}^{2}\right.$. $m\}_{k=1}^{\infty}$ such that $u_{n_{k}}^{2} \cdot m$ converges weakly to a probability measure $\nu=\phi_{0}^{2} \cdot m, \phi_{0} \in$ $\mathcal{D}(\mathcal{E}), \phi_{0} \geq 0$. Since the function $I_{\mathcal{E}}$ is lower semicontinuous by Proposition 2.4. $I_{\mathcal{E}}\left(\phi^{2} m\right) \leq \lambda_{2}$. Hence the function $\phi_{0}$ is just a ground state.

It follows from the inequality $\left\|\phi_{0}+\epsilon g\right\|_{\mathcal{E}}^{2} \geq \lambda_{2}\left\|\phi_{0}+\epsilon g\right\|_{2}^{2}$ holding for any $g \in \mathcal{D}(\mathcal{E})$ and for any $\epsilon>0$ that $\mathcal{E}\left(\phi_{0}, g\right)=\lambda_{2}\left(\phi_{0}, g\right)$. Hence $\alpha R_{\alpha-\lambda_{2}} \phi_{0}=\phi_{0}, \alpha>\lambda_{2}$, which implies that $\phi_{0}$ is strictly positive by irreducibility. 
To prove the uniqueness of the ground state, we introduce a closed symmetric form $\left(\mathcal{E}^{\phi_{0}}, \mathcal{D}\left(\mathcal{E}^{\phi_{0}}\right)\right)$ on $L^{2}\left(E ; \phi_{0}^{2} m\right)$ by

$$
\left\{\begin{array}{l}
\mathcal{E}^{\phi_{0}}(u, v)=\mathcal{E}\left(u \phi_{0}, v \phi_{0}\right)-\lambda_{2}\left(u \phi_{0}, v \phi_{0}\right) \\
\mathcal{D}\left(\mathcal{E}^{\phi_{0}}\right)=\left\{u \in L^{2}\left(E ; \phi_{0}^{2} \cdot m\right): u \phi_{0} \in \mathcal{D}(\mathcal{E})\right\}
\end{array}\right.
$$

Since $1 \in \mathcal{D}\left(\mathcal{E}^{\phi_{0}}\right), \mathcal{E}^{\phi_{0}}(1,1)=0$ and the associated resolvent $R_{\alpha}^{\phi_{0}}$ satisfies $R_{\alpha}^{\phi_{0}} f=$ $\phi_{0}^{-1} R_{\alpha-\lambda_{2}}\left(f \phi_{0}\right), \alpha>\lambda_{2}$, we see from the strict positivity of $\phi_{0}$ that $\left(\mathcal{E}^{\phi_{0}}, \mathcal{D}\left(\mathcal{E}^{\phi_{0}}\right)\right)$ is an irreducible recurrent Dirichlet form so that $f$ is constant whenever $f \in$ $\mathcal{D}\left(\mathcal{E}^{\phi_{0}}\right), \mathcal{E}^{\phi_{0}}(f, f)=0$. Let $\psi_{0}$ be another ground state. Then $\psi_{0}=f \phi_{0}$ with $f=\psi_{0} / \phi_{0} \in \mathcal{D}\left(\mathcal{E}^{\phi_{0}}\right), \mathcal{E}^{\phi_{0}}(f, f)=\mathcal{E}\left(\psi_{0}, \psi_{0}\right)-\lambda_{2}=0$, which yields that $f$ is constant and $\psi_{0}= \pm \phi_{0}$.

Let $\left\{u_{n}\right\}_{n=1}^{\infty} \subset \mathcal{D}(\mathcal{E})$ be a minimizing sequence in the proof of Lemma 2.6. We would like to emphasize that the tightness of $\left\{u_{n}^{2} \cdot m\right\}_{n=1}^{\infty} \subset \mathcal{P}$ and the lower semicontinuity of the function $I_{\mathcal{E}}$ with respect to the weak topology are used for the proof of the existence of the ground state, while the $\mathcal{E}_{1}$-weak compactness of $\left\{u_{n}\right\}_{n=1}^{\infty}$ in $\mathcal{D}(\mathcal{E})$ and the $\mathcal{E}_{1}$-weakly lower semicontinuity of $\mathcal{E}$ are usually used (e.g. [11, Section 11.1]). If $X$ is a Brownian motion on a Riemannian manifold or a symmetric $\alpha$-stable process, then we can show by employing the Rellich theorem that for $\mu \in \mathcal{K}_{\infty}$ the embedding of $\left(\mathcal{D}(\mathcal{E}), \mathcal{E}_{1}\right)$ to $L^{2}(E ; \mu)$ is compact ([16, Proposition 2], [17, Theorem 2.7]). Hence due to Lemma 2.2 and Proposition [2.4 we see that the resolvent $R_{\alpha}, \alpha>0$, is a compact operator on $L^{2}(E ; m)$ and the level set $\left\{\nu \in \mathcal{P}: I_{\mathcal{E}}(\nu) \leq \ell\right\}$ is a compact subset of $\mathcal{P}$. See [19] for another application of the existence of ground states.

\section{TightNess PROPERTY}

In this section, we will show that the tightness property implies a strong recurrence if $X$ is conservative and a fast explosion if $X$ is not conservative.

Lemma 3.1. An irreducible Borel right process $X$ with (2.2) satisfies one of the next two properties:

(a) (Conservative) $\mathbb{P}_{x}(\zeta<\infty)=0$ for all $x \in E$.

(b) (Explosive) $\quad \mathbb{P}_{x}(\zeta<\infty)>0$ for all $x \in E$.

Proof. Suppose $O:=\left\{x \in E: \mathbb{P}_{x}(\zeta<\infty)>0\right\}$ is not empty. Since $g(x):=\mathbb{P}_{x}(\zeta<$ $\infty$ ) is an excessive function, the set $O$ is a finely open set (e.g. 7, Theorem A.2.7]) and not $m$-polar. Indeed, if $O$ is $m$-polar, then $O$ is polar by the absolute continuity of the transition probability (2.2), and so $\mathbb{P}_{x}\left(\sigma_{O}<\infty\right)=0$ for all $x \in E$, which is contradictory to the fact that $\mathbb{P}_{x}\left(\sigma_{O}<\infty\right)>0$ for $x \in O$. (Note that [7, Theorem 4.1.2] holds for Borel right processes.) Since $O=\bigcup_{n=1}^{\infty} F_{n}, F_{n}=\{x \in$ $\left.E: \mathbb{P}_{x}(\zeta<\infty) \geq 1 / n\right\}$, some $F_{n}$ are not $m$-polar. Due to (2.2), we see from [7. Exercise 4.7.1] that $\mathbb{P}_{x}\left(\sigma_{F_{n}}<\infty\right)>0$ for all $x \in E$. Note that the set $F_{n}$ is finely closed and thus $X_{\sigma_{F_{n}}} \in F_{n}$ on $\left\{\sigma_{F_{n}}<\infty\right\}$. We then have

$$
\begin{aligned}
\mathbb{P}_{x}(\zeta<\infty) & =\mathbb{P}_{x}\left(\zeta<\infty, \sigma_{F_{n}}<\infty\right)+\mathbb{P}_{x}\left(\zeta<\infty, \sigma_{F_{n}}=\infty\right) \\
& \geq \mathbb{P}_{x}\left(\zeta\left(\theta_{\sigma_{F_{n}}}\right)<\infty, \sigma_{F_{n}}<\infty\right) \\
& =\mathbb{E}_{x}\left(\mathbb{P}_{X_{\sigma_{F_{n}}}}(\zeta<\infty) ; \sigma_{F_{n}}<\infty\right) \\
& \geq \frac{1}{n} \mathbb{P}_{x}\left(\sigma_{F_{n}}<\infty\right)>0 .
\end{aligned}
$$




\subsection{Conservative case.}

Lemma 3.2. Assume $X$ satisfies I-III. If $X$ is, in addition, conservative, then it is positively recurrent.

Proof. If $X$ is conservative, then the tightness property (III) implies that for any $\epsilon>0$, there exists a compact set $K$ such that $\inf _{x \in E} R_{1} 1_{K}(x) \geq 1-\epsilon$. Since the function $R_{1} 1_{K}$ is in $L^{1}(E ; m), m$ is finite, and thus $1 \in \mathcal{D}(\mathcal{E}), \mathcal{E}(1,1)=0$. Hence $X$ is positive recurrent ([7, Theorem 1.6.3]).

Remark 3.1. Suppose that $X$ is conservative and its semigroup satisfies the invariance of $C_{\infty}(E), p_{t}\left(C_{\infty}(E)\right) \subset C_{\infty}(E)$. Since

$$
\lim _{x \rightarrow \infty} R_{1} 1_{K^{c}}(x)=1-\lim _{x \rightarrow \infty} R_{1} 1_{K}(x)=1-0=1,
$$

$X$ does not have the tightness property.

Lemma 3.3. Assume $X$ satisfies (2.2). Then

$$
\sup _{x \in X} p_{t} 1(x)=\operatorname{ess}_{x \in X} p_{t} 1(x) \text {. }
$$

Proof. Let $M=\sup _{x \in X} p_{t} 1(x), \widetilde{M}=\operatorname{ess}_{\sup _{x \in X}} p_{t} 1(x)$. Suppose $M>\widetilde{M}$ and take $r$ so that $M>r>\widetilde{M}$. Since the function $p_{t} 1$ is excessive, the set $O=\{x \in$ $\left.X: p_{t} 1(x)>r\right\}$ is finely open and $m(O)=0$ by the definition of $\widetilde{M}$. Hence by Lemma 4.1.4 and Theorem 4.1.2 in [7], the set $O$ is polar and thus empty by the argument in the proof of Lemma 3.1. Therefore $p_{t} 1(x) \leq r$, which is contradictory to $M>r$.

Let us denote by $\left\|p_{t}\right\|_{p, p}$ the operator norm of $p_{t}$ from $L^{p}(X ; m)$ to $L^{p}(X ; m)$ and put

$$
-\lambda_{p}=\lim _{t \rightarrow \infty} \frac{1}{t} \log \left\|p_{t}\right\|_{p, p}, \quad 1 \leq p \leq \infty .
$$

$-\lambda_{p}$ is the long time exponential growth bound of the semigroup $\left\{p_{t}\right\}_{t \geq 0}$. The next theorem gives us a probabilistic interpretation of $\lambda_{\infty}$ (cf. [13]).

Theorem 3.4. Assume $X$ satisfies (2.2). Then

$$
\lambda_{\infty}=\sup \left\{\lambda \geq 0: \sup _{x \in E} \mathbb{E}_{x}\left(e^{\lambda \zeta}\right)<\infty\right\} .
$$

Proof. Let $\gamma$ be the right hand side of (3.4). Since for $\lambda<\gamma$,

$$
\left\|p_{t}\right\|_{\infty, \infty}=\sup _{x \in E} \mathbb{P}_{x}(t<\zeta) \leq e^{-\lambda t} \sup _{x \in E} \mathbb{E}_{x}\left(e^{\lambda \zeta}\right),
$$

$\gamma \leq \lambda_{\infty}$. In particular, if $\lambda_{\infty}=0$, then $\gamma=0$.

For $0<\lambda<\lambda_{\infty}$, let $p_{t}^{\lambda}=e^{\lambda t} p_{t}$. Then since

Hence

$$
\begin{gathered}
\lim _{t \rightarrow \infty} \frac{1}{t} \log \left\|p_{t}^{\lambda}\right\|_{\infty, \infty}=\lambda-\lambda_{\infty}<0, \\
\int_{0}^{\infty}\left\|p_{t}^{\lambda}\right\|_{\infty, \infty} d t=\int_{0}^{\infty} \sup _{x \in E} \mathbb{E}_{x}\left(e^{\lambda t} ; t<\zeta\right) d t<\infty .
\end{gathered}
$$

$$
\sup _{x \in E} \int_{0}^{\infty} \mathbb{E}_{x}\left(e^{\lambda t} ; t<\zeta\right) d t=\sup _{x \in E}\left(\frac{\mathbb{E}_{x}\left(e^{\lambda \zeta}\right)-1}{\lambda}\right)<\infty,
$$

and so $\gamma \geq \lambda_{\infty}$. 
Let us extend the resolvent operator; for $\lambda \geq 0$,

$$
R_{-\lambda} f(x)=\mathbb{E}_{x}\left(\int_{0}^{\infty} e^{\lambda t} f\left(X_{t}\right) d t\right) .
$$

We then see from (3.1) that for $\lambda>0$,

$$
\left\|R_{-\lambda}\right\|_{\infty, \infty}<\infty \Longleftrightarrow \sup _{x \in E} \mathbb{E}_{x}\left(e^{\lambda \zeta}\right)<\infty .
$$

It holds that if $\lambda_{\infty}>0$, then $\sup _{x \in E} \mathbb{E}_{x}\left(e^{\lambda_{\infty} \zeta}\right)=\infty$. Indeed, we see from (3.2) that if $\sup _{x \in E} \mathbb{E}_{x}\left(e^{\lambda_{\infty} \zeta}\right)<\infty$, then $\left\|R_{-\lambda_{\infty}}\right\|_{\infty, \infty}<\infty$. Noting that

$$
R_{-\lambda_{\infty}-\epsilon}=R_{-\lambda_{\infty}}+\epsilon R_{-\lambda_{\infty}}^{2}+\epsilon^{2} R_{-\lambda_{\infty}}^{3}+\cdots
$$

([10. III, §6]), we see that if $0<\epsilon<1 /\left\|R_{-\lambda_{\infty}}\right\|_{\infty, \infty}$, then $\left\|R_{-\lambda_{\infty}-\epsilon}\right\|_{\infty, \infty}<\infty$. Using (3.2) again, we have $\sup _{x \in E} \mathbb{E}_{x}\left(e^{\left(\lambda_{\infty}+\epsilon\right) \zeta}\right)<\infty$, which is contradictory to Theorem 3.4. Therefore, we have the next corollary.

Corollary 3.5. Suppose $\lambda_{\infty}>0$. Then

$$
\sup _{x \in E} \mathbb{E}_{x}(\exp (\lambda \zeta))<\infty \Longleftrightarrow \lambda<\lambda_{\infty} .
$$

Z.-Q. Chen [1, Theorem 4.1] proved:

Theorem 3.6. Suppose $X$ is irreducible and satisfies (2.2). If the measure $m$ belongs to $\mathcal{K}_{\infty}$, then $\lambda_{p}$ is independent of $p$.

Remark 3.7. The strong Feller property is not assumed in Theorem 3.6 while the $L^{p}$-independence is proven in [7, Theorem 6.4.3] under the assumptions (I)-(III). This extension is crucial when we show the $L^{p}$-independence for part processes of $X$. For a further extension of Theorem 3.6, see the recent papers [2], [3] of Z.-Q. Chen.

Combining Theorem 3.6 with Corollary 3.5, we have

Corollary 3.8. Suppose $X$ is irreducible and satisfies (2.2). If $m \in \mathcal{K}_{\infty}$ and $\lambda_{2}>0$, then

$$
\sup _{x \in E} \mathbb{E}_{x}(\exp (\lambda \zeta))<\infty \Longleftrightarrow \lambda<\lambda_{2} .
$$

Let $K \subset E$ be a compact set and $D:=K^{c}$, the complement of $K$. Let $X^{D}$ be the part process on $D$ :

$$
X^{D}=\left\{\begin{array}{l}
X_{t}, \quad t<\tau_{D}, \\
\Delta, \quad t \geq \tau_{D}, \quad \tau_{D}=\inf \left\{t \geq 0: X_{t} \notin D\right\} .
\end{array}\right.
$$

Define the (quasi-regular) Dirichlet form $\left(\mathcal{E}^{D}, \mathcal{D}\left(\mathcal{E}^{D}\right)\right)$ on $L^{2}(D ; m)$ by

$$
\left\{\begin{array}{l}
\mathcal{E}^{D}=\mathcal{E} \\
\mathcal{D}\left(\mathcal{E}^{D}\right)=\{u \in \mathcal{D}(\mathcal{E}): u=0 \text { q.e. on } K\} .
\end{array}\right.
$$

Then $\left(\mathcal{E}^{D}, \mathcal{D}\left(\mathcal{E}^{D}\right)\right)$ is the Dirichlet space generated by $X^{D}([7$, Theorem 4.4.3]).

Let $\lambda^{D}$ be the principal eigenvalue of the spectrum of $\left(\mathcal{E}^{D}, \mathcal{D}\left(\mathcal{E}^{D}\right)\right)$ :

$$
\lambda^{D}=\inf \left\{\mathcal{E}(u, u): u \in \mathcal{D}\left(\mathcal{E}^{D}\right), \int_{D} u^{2} d m=1\right\} .
$$

Lemma 3.9. Suppose that $X$ satisfies $I-I I I$ and is conservative. For any compact set $K$ with non-empty interior $K^{o}$, the principal eigenvalue $\lambda^{D}, D=K^{c}$, is positive. 
Proof. Let $\left\{\phi_{n}\right\}_{n=1}^{\infty} \subset \mathcal{D}\left(\mathcal{E}^{D}\right) \cap C_{0}(D)$ be an approximating sequence in (3.4) such that $\mathcal{E}\left(\phi_{n}, \phi_{n}\right) \rightarrow \lambda^{D}$. Let $\left\{\phi_{n_{k}}^{2} \cdot m\right\}_{k=1}^{\infty}$ be a subsequence of $\left\{\phi_{n}^{2} \cdot m\right\}_{n=1}^{\infty}$ weakly converging to $\phi_{0}^{2} \cdot m, \phi_{0} \in \mathcal{D}(\mathcal{E})$. Then

$$
1=\limsup _{k \rightarrow \infty} \int_{E \backslash K^{o}} \phi_{n_{k}}^{2} d m \leq \int_{E \backslash K^{o}} \phi_{0}^{2} d m,
$$

and thus $\phi_{0}$ equals $0, m$-a.e. on $K^{o}$. In particular, the function $\phi_{0}$ is not constant on $E$, because $m\left(K^{o}\right)>0$ by the assumption on $m$. Hence we have $\mathcal{E}\left(\phi_{0}, \phi_{0}\right)>0$. In fact, if $\mathcal{E}\left(\phi_{0}, \phi_{0}\right)=0$, then $\phi_{0}$ must be a constant by the irreducible recurrence of $(\mathcal{E}, \mathcal{D}(\mathcal{E}))([9$, Theorem 1.3]). We now conclude that

$$
\lambda^{D}=\liminf _{k \rightarrow \infty} \mathcal{E}\left(\phi_{n_{k}}, \phi_{n_{k}}\right) \geq \mathcal{E}\left(\phi_{0}, \phi_{0}\right)>0 .
$$

We write $\mathcal{K}_{\infty}\left(R_{1}\right)$ for $\mathcal{K}_{\infty}$ to express the dependence of the 1-resolvent. Let $R_{1}^{D}$ be the 1-resolvent of $X^{D}$. Denote by $m^{D}$ the restriction of $m$ to $D, m^{D}(\bullet)=$ $m(D \cap \bullet)$.

Lemma 3.10. Let $K$ be a compact set. Then $m^{D} \in \mathcal{K}_{\infty}\left(R_{1}^{D}\right), D=K^{c}$.

Proof. Let $\widetilde{K}$ and $\delta$ be a compact set and a positive constant in Definition 2.1. We can suppose that the interior of $\widetilde{K}$ contains $K$. Let $G$ be a relatively compact open set such that $K \subset G \subset \bar{G} \subset \widetilde{K}$ and $m(G \backslash K)<\delta$. Then $\widetilde{K} \cap G^{c}$ is a compact subset of $D$ and

$$
R_{1}^{D} 1_{\left(\widetilde{K} \cap G^{c}\right)^{c}}=R_{1}^{D} 1_{\widetilde{K}^{c} \cup(G \backslash K)} \leq R_{1} 1_{\widetilde{K}^{c}}+R_{1} 1_{G \backslash K} \leq \epsilon .
$$

Moreover, $R_{1}^{D} 1_{B} \leq R_{1} 1_{B}$ for any Borel set $B \subset \widetilde{K} \cap G^{c}$.

It follows from (2.7) that

$$
\int_{D} u^{2} d m=\int_{E} u^{2} 1_{D} d m \leq\left\|R_{1} 1_{D}\right\|_{\infty} \cdot\left(\mathcal{E}(u, u)+\int_{E} u^{2} d m\right), \quad u \in \mathcal{D}\left(\mathcal{E}^{D}\right),
$$

and thus

$$
1 \leq\left\|R_{1} 1_{D}\right\|_{\infty} \cdot\left(\lambda^{D}+1\right) .
$$

The tightness property implies that there exists a sequence $\left\{K_{n}\right\}_{n=1}^{\infty}$ of compact sets such that $\bigcup_{n=1}^{\infty} K_{n}=E$ and $\left\|R_{1} 1_{K_{n}^{c}}\right\|_{\infty} \rightarrow 0$ as $n \rightarrow \infty$. Hence we see from (3.5) that for $D_{n}=K_{n}^{c}$,

$$
\lambda^{D_{n}} \uparrow \infty \text { as } n \rightarrow \infty .
$$

Note that if $X$ is conservative, then the lifetime of $X^{D}$ equals the hitting time of $K$. Combining Lemma 3.10 with Corollary 3.8, we know that if $X^{D_{n}}$ is irreducible, then

$$
\sup _{x \in D_{n}} \mathbb{E}_{x}\left(\exp \left(\gamma \sigma_{K_{n}}\right)\right)<\infty \Longleftrightarrow \gamma<\lambda^{D_{n}}
$$

Note that

$$
\sup _{x \in D} \mathbb{E}_{x}\left(\exp \left(\sigma_{K}\right)\right)=\sup _{x \in E} \mathbb{E}_{x}\left(\exp \left(\sigma_{K}\right)\right)
$$


Indeed, let $x_{0} \in K \backslash K^{r}$, where $K^{r}$ is the regular set of $K, \mathbb{P}_{x}\left(\sigma_{K}=0\right)=1$. Then since

$$
\begin{aligned}
\mathbb{E}_{x_{0}}\left(\exp \left(\sigma_{K}\right)\right) & =\mathbb{E}_{x_{0}}\left(\exp \left(\sigma_{K}\right) ; X_{t} \in K\right)+\mathbb{E}_{x_{0}}\left(\exp \left(\sigma_{K}\right) ; X_{t} \in D\right) \\
& \leq e^{t} \mathbb{P}_{x_{0}}\left(X_{t} \in K\right)+e^{t} \mathbb{E}_{x_{0}}\left(\exp \left(t+\sigma_{K}\left(\theta_{t}\right)\right) ; X_{t} \in D\right) \\
& \leq e^{t} \mathbb{P}_{x_{0}}\left(X_{t} \in K\right)+e^{t} \mathbb{E}_{x_{0}}\left(\mathbb{E}_{X_{t}}\left(\exp \left(\sigma_{K}\right)\right) ; X_{t} \in D\right) \\
& \leq e^{t} \mathbb{P}_{x_{0}}\left(X_{t} \in K\right)+e^{t} \sup _{x \in D} \mathbb{E}_{x}\left(\exp \left(\sigma_{K}\right)\right)
\end{aligned}
$$

and

$$
\mathbb{P}_{x_{0}}\left(X_{t} \in K\right) \leq \mathbb{P}_{x_{0}}\left(\sigma_{K} \leq t\right) \longrightarrow 0 \text { as } t \downarrow 0,
$$

we have (3.8) and thus

$$
\sup _{x \in E} \mathbb{E}_{x}\left(\exp \left(\gamma \sigma_{K_{n}}\right)\right)<\infty \Longleftrightarrow \gamma<\lambda^{D_{n}} .
$$

Hence we have from (3.6) and (3.9) the following:

Lemma 3.11. Suppose that $X$ satisfies $I-I I I$ and is conservative. If there exists an increasing sequence $\left\{K_{n}\right\}_{n=1}^{\infty}$ of compact sets such that $\bigcup_{n=1}^{\infty} K_{n}=E$ and $X^{D_{n}}$, $D_{n}=K_{n}^{c}$, are irreducible, then $X$ has the following property:

For any $\gamma>0$ there exists a compact set $K$ such that

$$
\sup _{x \in E} \mathbb{E}_{x}\left(\exp \left(\gamma \sigma_{K}\right)\right)<\infty .
$$

Property $(\mathrm{H})$ is said to be a uniform hyper-exponential recurrence ([21]). We will give sufficient conditions for the part process $X^{D}$ being irreducible (Lemma 4.2 , Lemma 4.3 .

Noting that

$$
p_{t}(x, U)=0 \text { for } \forall t>0 \Longleftrightarrow \mathbb{P}_{x}\left(\sigma_{U}<\infty\right)=0,
$$

we see that if $X$ is irreducible, the semigroup $\left\{p_{t}\right\}_{t \geq 0}$ is topological transitive; that is, for all non-empty open sets $U$ and $x \in E$, there exists $t>0$ such that $p_{t}(x, U)>0$. Therefore, Theorem 1.2 in $\mathrm{Wu}$ [21] leads us to:

Theorem 3.12. Suppose $X$ satisfies $I-I I I$ and is conservative. If there exists an increasing sequence $\left\{K_{n}\right\}_{n=1}^{\infty}$ of compact sets such that $\bigcup_{n=1}^{\infty} K_{n}=E$ and $X^{D_{n}}$, $D_{n}=K_{n}^{c}$, are irreducible, then the uniform large deviation principle holds: for each open set $G$ of $\mathcal{P}$,

$$
\liminf _{t \rightarrow \infty} \frac{1}{t} \log \inf _{x \in E} \mathbb{P}_{x}\left(L_{t} \in G\right) \geq-\inf _{\mu \in G} I_{\mathcal{E}}(\mu) .
$$

Example 3.1 (One-dimensional diffusion processes). Let us consider a one-dimensional diffusion process $X=\left(X_{t}, \mathbb{P}_{x}, \zeta\right)$ on an open interval $I=\left(r_{1}, r_{2}\right)$ such that $\mathbb{P}_{x}\left(X_{\zeta-}=r_{1}\right.$ or $\left.r_{2}, \zeta<\infty\right)=\mathbb{P}_{x}(\zeta<\infty), x \in I$, and $\mathbb{P}_{a}\left(\sigma_{b}<\infty\right)>0$ for any $a, b \in I$. The diffusion $X$ is symmetric with respect to its canonical measure $m$ and satisfies I and II. The boundary point $r_{i}$ of $I$ is classified into four classes: regular boundary, exit boundary, entrance boundary, and natural boundary ([8, Chapter 5]):

(a) If $r_{2}$ is a regular or exit boundary, then $\lim _{x \rightarrow r_{2}} R_{1} 1(x)=0$.

(b) If $r_{2}$ is an entrance boundary, then $\lim _{r \rightarrow r_{2}} \sup _{x \in\left(r_{1}, r_{2}\right)} R_{1} 1_{\left(r, r_{2}\right)}(x)=0$.

(c) If $r_{2}$ is a natural boundary, then $\lim _{x \rightarrow r_{2}} R_{1} 1_{\left(r, r_{2}\right)}(x)=1$ and thus $\sup _{x \in\left(r_{1}, r_{2}\right)} R_{1} 1_{\left(r, r_{2}\right)}(x)=1$. 
Therefore, tightness property III is fulfilled if and only if no natural boundaries are present. As a corollary of equation (3.6), if $r_{2}$ is an entrance boundary, for any $\lambda>0$ there exists $r_{1}<r<r_{2}$ such that

$$
\sup _{x>r} \mathbb{E}_{x}\left(\exp \left(\lambda \sigma_{r}\right)\right)<\infty
$$

where $\sigma_{r}$ is the first hitting time of $\{r\}$. Therefore, if both the boundaries are entrance, then the uniform large deviation holds. Let $p_{t}(x, y)$ be the transition probability density of $X$. We see from [15] that if $X$ is uniformly ergodic, that is, there exists a positive constant $M$ such that

$$
p_{1}(x, z) \leq M \cdot p_{1}(y, z) \text { for any } x, y, z \in I,
$$

then it satisfies the uniform large deviation principle. Nevertheless, we do not know that a one-dimensional diffusion process with entrance boundaries always satisfies the uniform ergodicity.

We see from [8] that the Ornstein-Uhlenbeck process on the one-dimensional space $\mathbb{R}$ has natural boundaries and its semigroup keeps $C_{\infty}(\mathbb{R})$ invariant. Hence due to Remark 3.1, we see that the Ornstein-Uhlenbeck process does not possess the tightness property. Moreover, it is known in 21 that the Ornstein-Uhlenbeck process does not satisfy the uniform large deviation, while it satisfies the locally uniform large deviation.

\section{IRREDUCIBILITY OF PART PROCESSES}

In this section, we consider conditions for part processes being irreducible. If $X$ is a diffusion process generated by a locally uniform elliptic operator, then its part process on a domain is irreducible ([7, Corollary 4.6.4, Example 4.6.1]). More generally, we have:

Lemma 4.1. Assume $R_{1} 1 \in C_{\infty}(E)$. If $D \subset E$ is a connected open set, then $X^{D}$ satisfies I-III.

Proof. By the assumption, $X$ is a doubly Feller process; that is, it satisfies the strong Feller property and the invariance of $C_{\infty}(E)$. We then know from Chung [4] that $X^{D}$ has the strong Feller property. Hence Exercise 4.6.3 in [7] leads us to this lemma.

We next treat jump processes. Let $\left(N(x, d y), H_{t}\right)$ be a Lévy system of $X$. We make the next assumption:

$$
\text { (J) } \begin{cases}(i) & \text { If } m(B)>0, \text { then } N(x, B)>0 \text { for any } x \in E . \\ (\text { ii }) & \left\{x \in E: \mathbb{P}_{x}\left(\inf \left\{t>0: H_{t}>0\right\}=0\right)=1\right\}=E .\end{cases}
$$

Lemma 4.2. Assume $(\mathrm{J})$. Then for any compact set $F \subset D$ with $m(F)>0$, $\mathbb{P}_{x}\left(\sigma_{F}<\tau_{D}\right)>0$.

Proof. Let $x \notin D \backslash F$ and take $r>0$ such that $B(x, r) \cap F=\emptyset$. Then

$$
\mathbb{E}_{x}\left(\sum_{0<s<\tau_{D}} 1_{B(x, r)}\left(X_{s-}\right) 1_{F}\left(X_{s}\right)\right)=\mathbb{E}_{x}\left(\int_{0}^{\tau_{D}} 1_{B(x, r)}\left(X_{s}\right) N\left(X_{s}, F\right) d H_{s}\right) .
$$

The right hand side is positive by the assumption, which leads us to the lemma.

Lemma 4.3. Assume $(\mathrm{J})$. Let $K \subset D$ be a set with $m(K)>0$. Then $R_{1}^{D}(x, K)>0$ for any $x \in D$. 
Proof. Since

$$
\int_{D} R_{1}^{D}(x, K) d m=\int_{D} R_{1}^{D} 1(x) 1_{K}(x) d m>0,
$$

the set $\left\{x \in D: R_{1}^{D}(x, K)>0\right\}$ is of positive $m$-measure. Take a compact set $F$ such that $F \subset\left\{x \in D: R_{1}^{D}(x, K)>0\right\}$ and $m(F)>0$. Then

$$
\begin{aligned}
R_{1}^{D}(x, K) & =\mathbb{E}_{x}\left(\int_{0}^{\tau_{D}} e^{-t} 1_{K}\left(X_{t}\right) d t\right) \geq \mathbb{E}_{x}\left(\int_{\sigma_{F}}^{\tau_{D}} e^{-t} 1_{K}\left(X_{t}\right) d t ; \sigma_{F}<\tau_{D}\right) \\
& =\mathbb{E}_{x}\left(e^{-\sigma_{F}} R_{1}^{D}\left(X_{\sigma_{F}}, K\right) ; \sigma_{F}<\tau_{D}\right) .
\end{aligned}
$$

The right hand side is positive by Lemma 4.2 .

4.1. Explosive case. If $X$ is explosive, then the principal eigenvalue $\lambda$ is positive. Indeed, if the ground state $\phi_{0}$ satisfies $\mathcal{E}\left(\phi_{0}, \phi_{0}\right)=0$, then $\phi_{0}=0$ by transience. It follows from Corollary 3.8 that

$$
\sup _{x \in E} \mathbb{E}_{x}(\exp (\gamma \zeta))<\infty \Longleftrightarrow \gamma<\lambda_{2} .
$$

In particular, property (b) in Lemma 3.1 can be strengthened to $\mathbb{P}_{x}(\zeta<\infty)=1$.

\section{REFERENCES}

[1] Zhen-Qing Chen, Gaugeability and conditional gaugeability, Trans. Amer. Math. Soc. 354 (2002), no. 11, 4639-4679 (electronic), DOI 10.1090/S0002-9947-02-03059-3. MR.1926893 (2003i:60127)

[2] Zhen-Qing Chen, Uniform integrability of exponential martingales and spectral bounds of non-local Feynman-Kac semigroups, Stochastic analysis and applications to finance, Interdiscip. Math. Sci., vol. 13, World Sci. Publ., Hackensack, NJ, 2012, pp. 55-75. MR2986841

[3] Z.-Q. Chen, $L^{p}$-independence of spectral bounds of generalized non-local Feynman-Kac semigroups, J. Funct. Anal. 262 (2012), 4120-4139. MR2899989

[4] K. L. Chung, Doubly-Feller process with multiplicative functional, Seminar on stochastic processes, 1985 (Gainesville, Fla., 1985), Progr. Probab. Statist., vol. 12, Birkhäuser Boston, Boston, MA, 1986, pp. 63-78. MR896735(88k:60128)

[5] E. B. Davies, $L^{1}$ properties of second order elliptic operators, Bull. London Math. Soc. 17 (1985), no. 5, 417-436, DOI 10.1112/blms/17.5.417. MR806008 (87g:58126)

[6] M. D. Donsker and S. R. S. Varadhan, Asymptotic evaluation of certain Markov process expectations for large time. I. II, Comm. Pure Appl. Math. 28 (1975), 1-47; ibid. 28 (1975), 279-301. MR0386024 (52 \#6883)

[7] Masatoshi Fukushima, Yoichi Oshima, and Masayoshi Takeda, Dirichlet forms and symmetric Markov processes, Second revised and extended edition, de Gruyter Studies in Mathematics, vol. 19, Walter de Gruyter \& Co., Berlin, 2011. MR2778606 (2011k:60249)

[8] Kiyoshi Itô, Essentials of stochastic processes, Translations of Mathematical Monographs, vol. 231, American Mathematical Society, Providence, RI, 2006. Translated from the 1957 Japanese original by Yuji Ito. MR2239081 (2007i:60001)

[9] N. Kajino, Equivalence of recurrence and Liouville property for symmetric Dirichlet forms, preprint (2010).

[10] Tosio Kato, Perturbation theory for linear operators, Classics in Mathematics, SpringerVerlag, Berlin, 1995. Reprint of the 1980 edition. MR1335452 (96a:47025)

[11] Elliott H. Lieb and Michael Loss, Analysis, 2nd ed., Graduate Studies in Mathematics, vol. 14, American Mathematical Society, Providence, RI, 2001. MR 1817225 (2001i:00001)

[12] Zhi Ming Ma and Michael Röckner, Introduction to the theory of (nonsymmetric) Dirichlet forms, Universitext, Springer-Verlag, Berlin, 1992. MR.1214375 (94d:60119)

[13] Sadao Sato, An inequality for the spectral radius of Markov processes, Kodai Math. J. 8 (1985), no. 1, 5-13, DOI 10.2996/kmj/1138036992. MR776702 (86h:60144)

[14] Peter Stollmann and Jürgen Voigt, Perturbation of Dirichlet forms by measures, Potential Anal. 5 (1996), no. 2, 109-138, DOI 10.1007/BF00396775. MR1378151 (97e:47065) 
[15] D. W. Stroock, An introduction to the theory of large deviations, Universitext, SpringerVerlag, New York, 1984. MR755154(86h:60067a)

[16] M. Takeda, Branching Brownian motions on Riemannian manifolds: expectation of the number of branches hitting closed sets, Potential Anal. 27 (2007), no. 1, 61-72, DOI 10.1007/s11118-007-9039-3. MR2314189 (2008g:60263)

[17] Masayoshi Takeda, Large deviations for additive functionals of symmetric stable processes, J. Theoret. Probab. 21 (2008), no. 2, 336-355, DOI 10.1007/s10959-007-0111-0. MR.2391248 (2009k:60175)

[18] Masayoshi Takeda, A large deviation principle for symmetric Markov processes with Feynman-Kac functional, J. Theoret. Probab. 24 (2011), no. 4, 1097-1129, DOI 10.1007/s10959-010-0324-5. MR2851247

[19] M. Takeda and Y. Tawara, A large deviation principle for symmetric Markov processes normalized by Feynman-Kac functionals, to appear in Osaka J. Math.

[20] Yoshihiro Tawara, $L^{p}$-independence of spectral bounds of Schrödinger-type operators with non-local potentials, J. Math. Soc. Japan 62 (2010), no. 3, 767-788. MR2648062 (2012a:60208)

[21] Liming $\mathrm{Wu}$, Some notes on large deviations of Markov processes, Acta Math. Sin. (Engl. Ser.) 16 (2000), no. 3, 369-394, DOI 10.1007/PL00011549. MR1787093 (2001i:60048)

Mathematical Institute, Tohoku University, Aoba, Sendai, 980-8578, Japan

E-mail address: takeda@math.tohoku.ac.jp 